\title{
SORÇÃO DE FÓSFORO EM FUNÇÃO DO TEOR INICIAL E DE SISTEMAS DE MANEJ O DE SOLOS ${ }^{(1)}$
}

\author{
D. S. RHEINHEIME ${ }^{(2)}$, I. ANGHINONI(3) \& E. CONTE ${ }^{(4)}$
}

\begin{abstract}
RESUMO
A relação entre o $\mathbf{P}$ sorvido/ $\mathbf{P}$ solução do solo pode ser influenciada pelo teor de $\mathrm{P}$ previamente sorvido e pelos sistemas de manejo. Este trabalho teve o objetivo de avaliar as modificações na capacidade de sorção máxi ma $\left(P_{\max }\right)$ e na constante relacionada com a energia de ligação de $P$ com os colóides $(k)$ da isoterma de Langmuir, provocadas (a) pela inclusão do teor de $\mathbf{P}$ previamente sorvido $\left(P_{\text {pre }}\right)$ e (b) por sistemas de manejo de solos. Coletaram-se, em maio de 1997, amostras de solo (Latossolo Vermelho distroférrico típico, Latossolo Vermelho distrófico típico e Argissolo Vermelho distrófico típico), em três camadas (0-2,5, 2,5-7,5 e 7,5-17,5 cm), de três experimentos instalados a partir de 1979, envolvendo os sistemas plantio di reto e cultivo convencional com diferentes sucessões de cultura. $\mathbf{O}$ solo foi equilibrado com oito concentrações de $\mathbf{P}$ em solução de $\mathrm{CaCl}_{2}$ 0,001 mol L-1. Após a agitação por $16 \mathrm{~h}$, avaliou-se a concentração de $\mathbf{P}$ no sobrenadante. Os dados de $\mathbf{P}$-sorvido e $\mathrm{P}$-solução foram ajustados à equação de Langmuir, obtendo-se a $P_{\max } \mathbf{e}$ a $k$, considerando ou não o $P_{\text {pre: }} \mathbf{A}$ inclusão do $P_{\text {pre }}$ no modelo de Langmuir aumenta, em média, 2,9 vezes o valor da $k$ e não afeta a $P_{\text {max }}$, no LVdf, e aumenta em todas as camadas, no LVd, e na camada superficial, no PVd. A $\mathbf{P}_{\max }$ é pouco influenciada pelos métodos de preparo do solo, sucessões de culturas e camadas amostradas.
\end{abstract}

Termos para indexação: adubação fosfatada, isoterma de Langmuir, preparo do solo.

(1) Trabal ho realizado com apoio financeiro do PRONEX-FINEP e FAPE RGS. Recebido para publicação em junho de 2001 e aprovado em outubro de 2002.

(2) Professor do Departamento de Solos, Universidade Federal de Santa Maria - UFSM. Caixa Postal 221, CEP 97119-900 Santa Maria (RS). E-mail: Danilo@ccr.ufsm.br

(3) Professor do Departamento de Solos, Universidade Federal do Rio Grande do Sul - UFRGS. Caixa Postal 776, CEP 91540-000 Porto Alegre (RS). Bolsista do CNPq.

(4) Engenheira-Agrônoma, Mestre em Ciência do Solo, Departamento de Solos, UFRGS. 


\title{
SUMMARY: PHOSPHORUS SORPTION IN SOIL IN RELATION TO THE INITIAL CONTENT AND SOIL MANAGEMENT
}

\begin{abstract}
Thesorbed P/ P in solution relation of thesoil may beaffected by the previously sorbed $P\left(P_{\text {pre }}\right)$ content and by soil management systems. Theobjectiveof this study was to evaluate modifications in the maximum $P$ sorption capacity $\left(P_{\max }\right)$ and the constant related to the colloid linkage energy $(k)$ of the Langmuir isotherm caused $(a)$ by theincl usion of $P_{\text {pre }}$ and (b) by different soil tillagemethods and crop sequences. In May 1997, samples of two Oxisols (Very clayey Hapludox and Clayey Hapludox) and an UItisol (Paleudult) were col lected in three soil layers $(0-2.5,2.5-7.5$ and $7.5-17.5 \mathrm{~cm}$ ) of three fiel d experiments, installed from 1979 on, under conventional and no tillage systems with different crop sequences. The samples were equilibrated with eight $\mathrm{P}$ levels in $0.001 \mathrm{~mol} \mathrm{~L}^{-1} \mathrm{CaCl}_{2}$ solution. After $16 \mathrm{~h}$ of shaking, theP content in thesupernatant was determined. Thesorbed and solution P data were adjusted to the Langmuir equation, and $\mathrm{P}_{\max }$ and $\mathrm{k}$ parameters determined by considering $\mathrm{P}_{\text {pre }}$ or not. On average, theinclusion of $\mathrm{P}_{\text {pre }}$ in the Langmuir equation increased the $k$ value 2.9 times, but did not affect $P_{\max }$ in the very clayey Hapludox, which increased in all soil layers of theclayey Hapludox and in the surfacelayer of the Pal eudult. $P_{\max }$ was little affected by the soil tillage methods, crop sequence or soil layers.
\end{abstract}

Index terms: phosphatefertilization, Langmuir isotherm, soil tillage.

\section{INTRODUÇÃO}

A sorção é entendida como a transferência reversível de íons entre a solução e a fase sólida do solo (McBride, 1994). Compreende a adsorção por forças físicas (complexo de esfera externa) e por forças químicas ou específicas (complexo de esfera interna), a preci pitação ea polimerização. A maioria dos ânions é adsorvida sobre a superfície dos minerais, podendo o íon fosfato ser adsorvido especificamente por óxidos de ferro, alumínio e manganês, nas arestas das argilas silicatadas e nos aluminossilicatos amorfos, com a participação dos grupos $\mathrm{OH}^{-}$ou $\mathrm{OH}_{2}{ }^{+}$monocoordenados, os quais facilitam a protonação e as reações de troca de ligantes. Nelas ocorre a liberação de $\mathrm{OH}^{-}$para a solução do solo, com alto grau de especificidade e tendência à não-reversibilidade, o que aumenta as cargas superficiais negativas e abaixa o ponto de carga zero (McBride, 1994).

Segundo Parfitt (1989), a adsorção de fósforo ocorre na forma binucleada e em três estádios, conforme o tempo de reação: no primeiro, a reação dá-se rápida e fortemente em sítios muito reativos, onde silicatos, $\mathrm{OH}^{-} \mathrm{e} \mathrm{OH}_{2}{ }^{+}$, são trocados por fosfato; no segundo, el é adsorvido em sítios menos reativos e, no terceiro, a longo prazo, migra, via porosidade, para os lóbulos internos dos cristais, podendo, também, ser considerado como precipitação.

A magnitude da adsorção depende da quantidade de constituintes com capacidade de sorver moléculas neutras ou carregadas el etricamente. Nos solos de regiões tropicais e subtropicais, os óxidos de ferro mal cristalizados, a quantidade de alumínio substituindo ferro, e em menores quantidades, os óxidos bem cristalizados e a caulinita são os principais responsáveis por esse fenômeno (Fontes \& Weed, 1996).

A capacidade máxima de sorção de fósforo, $\mathrm{P}_{\max }$, correlaciona-se com os teores de $\mathrm{Fe}_{0}+\mathrm{Al}_{\mathrm{O}}$ (F reese et al., 1992; Scheinost \& Schwertmann, 1995). No entanto, no valor de $P_{\max }$, não está incluída a quantidade de fósforo previamentesorvida pelo solo, $\mathrm{P}_{\text {pre, }}$ que pode variar de 17 a $66 \%$ do $\mathrm{P}_{\max }$ (Freeseet al., 1992). Adições de fertilizantes fosfatados (maior $P_{\text {pre }}$ ) proporcionam maior cobertura dos sítios de sorção, saturando-os e diminuindo a variação da energia livre proporcional mente ao recobrimento da superfície (Fox \& Kamprath, 1970; Ryden et al., 1977; Barrow, 1978). I sso aumenta a dessortividade e diminui a relação sorvido/solução (Hartikainen, 1991; Raven \& Hossner, 1993; Tol ner \& Füleky, 1995), o que pode causar erros de interpretação nas isotermas de adsor ção.

Dados de Oloya \& Logan (1980) e Guertal et al . (1991) mostram que, em solos sob sistema plantio direto, ocorre menor adsorção na camada de $0-2 \mathrm{~cm}$, independentemente da dose total defosfato aplicada, demonstrando um decréscimo na retenção. A quantidade do fósforo removida pela extração seqüencial com resina foi mais rápida e em maior quantidade na camada superficial, em relação às demais camadas. Após a extração seqüencial, a capacidade de sorção de fósfor o foi mai or para todas as profundidades, sobretudo para a camada superficial. Então, a menor sorção do fosfato adicionado deve-se à maior quantidade de fósforo que já estava adsorvido aos colóides (saturação dos sítios). O maior teor de matéria orgânica da camada superficial no sistema plantio direto pode diminuir a capacidade de adsorção máxima de fosfato e a 
energia de ligação do fosfato aos grupos funcionais dos colóides inorgânicos do sol o. Os ânions deácidos orgânicos podem complexar Fe eAl e, ou, seadsorver à superfície de óxidos, bloqueando os sítios de adsorção de fósforo (Sibanda \& Young, 1986; Mesquita \& Torrent, 1993).

Este trabal ho teve o objetivo de estimar o efeito do fósforo previamente sorvido na determinação da capacidade de sorção máxima ena energia de sorção de fósforo influenciado por sistemas de manejo do solo.

\section{MATERIAL E MÉTODOS}

As amostras de solo utilizadas neste trabal ho foram coletadas de três experimentos de longa duração, instalados sobretrês tipos de solos. Foram utilizados experimentos em que se estudaram os efeitos demétodos de preparo do soloe de sistemas de sucessão de culturas na recuperação da qualidade do solo.

O primeiro experimento foi instalado em 1979 num Latossolo Vermel ho distroférrico típico muito argil oso, substrato basalto (LVdf - Rhodic Hapludox), no Centro de Atividades Agrícolas e Florestais da Cooperativa Tritícola de Santo Ângelo (RS). A área tinha sido anteriormente cultivada por 15 anos em cultivo convencional eapresentava avançado estado de degradação física.

Na instalação do experimento, em 1985, e, posteriormente, em 1992 aplicou-se cal cário para el evar o pH até6,0 (método SMP). A adubação usada a partir da instalação do experimento seguiu a recomendação técnica específica a cada cultura.

As amostras de sol o foram col etadas nas parcelas do sistema plantio direto (SPD) e nas do cultivo convencional (SCC), com as sucessões de culturas aveia (Avena strigosa Screb)/milho (Zea mays L.) e trigo (Triticum aestivum L.)/soja (Glycine max (L.) Merrill). NoSPD, col etaram-se amostras, também, das parcelas com a rotação envolvendo as culturas: trigo, soja, tremoço (L upinus angustifoliumL.), milho, sorgo (Sorghum bicolor L.) e aveia preta+trevo (Trifolium repens L.). As amostras de solo foram coletadas nas parcelas que nunca receberam adubação nitrogenada, na expectativa de que maior quantidade de fósforo acumular-se-ia no solo, uma vez quea exportação pelas col heitas era menor. Maiores detal hes podem ser obtidos em Dalla Rosa et al. (1991).

O segundo experimento foi instalado em 1983 num L atossolo Vermelho distrófico típico argiloso, substrato basal to (LVd - Rhodic Hapludox), no Centro Nacional dePesquisa doTrigo- EMBRAPA, em Passo Fundo (RS). As amostras foram coletadas nos tratamentos SPD eSCC enos blocos cultivados com sorgo esoja, deuma seqüência deculturas alternadas no tempo, envolvendo vica (Vicia sativa L.), milho, sorgo, aveia preta, soja e cevada (Hordeum vulgare L.). O calcário foi aplicado no experimento ereaplicado em 1989, sendo incorporado ao solo na dose recomendada para elevação do pH até6,0. A adubação usada seguiu as recomendações técnicas. Maiores detal hes podem ser obtidos em Selles et al. (1997).

O terceiro experimento foi instalado em 1985 num Argissolo Vermel ho distrófico franco-argilosoarenoso (PVd - Rhodic Paleudult) degradado fisicamente, em Eldorado do Sul (RS), pelo Departamento de Sol os da Universidade Federal do RioGrandedoSul. Na época, ocalcáriofoi incorporado ao solo e as demais aplicações foram feitas na superfície. O solo foi amostrado nos tratamentos SPD e SCC nas sucessões de culturas aveia/milho e aveia + vica/milho + caupi (Vigna unguiculata subs unguiculata (L.) Walp). Maiores detal hes podem ser obtidos em Amado et al. (1999).

Em maio de 1997, logo após o preparo, coletaramse amostras de sol o nos três experimentos, em duas repetições de cada tratamento, nas profundidades de $0-2,5 ; 2,5-7,5$ e 7,5-17,5 cm. Cada amostra foi composta pela homogeneização de duas subamostras oriundas de trincheiras de $50 \times 10 \times 17,5 \mathrm{~cm}$, coletadas perpendicularmente às linhas de semeadura. O solo foi seco ao ar e peneirado em malha de $1 \mathrm{~mm}$.

Para avaliar a sorção de fósforo, 3,00 g de solo foram equilibrados com $30 \mathrm{~mL}$ de solução de $\mathrm{CaCl}_{2}$ $0,001 \mathrm{~mol} \mathrm{~L}^{-1}$, com oito concentrações de fósforo $(0$, $20,40,80,160,320,640$ e $1.280 \mathrm{mg} \mathrm{L}^{-3}$ ), para os Latossolos, eas sete primeiras, para oArgissolo. Após a agitação por $16 \mathrm{~h}$ no"end - over - end", centrifugouse (8.000 rpm por $10 \mathrm{~min}$ ) e avaliou-se, no sobrenadante, a concentração de fósforo de acordo com o método Murphy \& Riley (1962). O fósforo sorvido foi obtido pela diferença entre a quantidade adicionada e a recuperada na solução de equilíbrio. Os dados de P-sorvido e P-solução foram ajustados à equação de Langmuir, $P_{\text {sorvido }}=k$. $P_{\max }$. $P_{\text {soluçãol }}$ $\left.1+k . P_{\text {solucãoo }}\right)$, em que $k=$ constanterelacionada com a energia de sorção, $\mathrm{P}_{\max }=$ capacidade de sorção máxima e $P_{\text {solução }}=$ concentração na solução, considerando ou não a quantidade de fósforo previamente sorvida pelo solo $\left(\mathrm{P}_{\text {pre }}\right)$ (Tolner \& Füleky, 1995). Considerou-se como $P_{\text {pre }}$ a quantidade máxima de fósforo dessorvido com extrações sucessivas com resina em membrana (Rheinheimer et al., 2000), o qual foi somado ao fósforo sorvido. Calculou-sea contribuição percentual do $P_{\text {pre }}$ no $P_{\max }$. O teor de carbono orgânico foi determinado por digestão úmida.

Os valores de $\mathrm{P}_{\max }$ e de $\mathrm{k}$ foram submetidos à análise de variância a $5 \%$ designificância, enquanto as médias dos tratamentos de preparo do solo, sucessão de culturas e profundidade de amostragem foram comparadas pelo teste Tukey $(p<0,05)$. E fetuaram-se correlações a $P_{\max }$ e a $k$ com o teor de carbono orgânico. 


\section{RESULTADOS E DISCUSSÃO}

\section{Alterações na relação fósforo sorvido/fósforo solução com a inclusão do $\mathbf{P}_{\text {pre }}$}

No LVdf, sem considerar o $P_{\text {pre, }}$ o solo sob SPD manteve maiores teores de $P$ na solução do que no solo sob SCC, enquanto, com a inclusão do $\mathrm{P}_{\text {pre, }}$ os teores de $\mathrm{P}$ solução foram semelhantes entre os métodos de preparo do solo. No LVd, para todos os valores de P sorvido, o sol o sob SPD manteve mais altos os teores de $\mathrm{P}$ na solução de equilíbrio. I sso poderia ser interpretado como sendo um efeito da competi ção exercida pela matéria orgânica, uma vez que as maiores diferenças ocorreram na camada superficial, justamente onde os seus teores são mais el evados. No entanto, a inclusão dos val ores de $P_{\text {pre }}$ na isoterma de Langmuir resultou em teores sorvidos maiores no solo sob SPD do que sob SCC, especialmente para doses baixas de $\mathrm{P}$ aplicado. No PVd, a quantidade de P sorvido foi levementemenor no sol o sob SPD do que sob SCC até $7,5 \mathrm{~cm}$. Com a inclusão do $\mathrm{P}_{\text {pre, }}$ esse comportamento inverteu-se somente para a camada superficial, onde se acumulou o P adicionado como fertilizante (Rheinheimer \& Anghinoni, 2001). Na camada de 2,5-7,5 cm, com a sucessão aveia/mil ho, e na camada de 7,5-17,5 cm, com ambas as sucessões de culturas, observou-se maior sorção de P no solo sob SCC do que sob SPD, pelo menos em doses mais baixas, o que está de acordo com os maiores teores de $\mathrm{P}_{\text {pre }}$ (Figuras 1, 2 e 3).

A capacidade máxima de sor ção de fósforo $\left(P_{\max }\right)$ do LVdf não foi alterada com a inclusão do $\mathrm{P}_{\text {pre }}$ no ajuste da isoterma de Langmuir (Quadro 1). Para o LVd, a inclusão do $P_{\text {pre }}$ aumentou a $P_{\max }$ no SPD de 937 para $1.021 \mathrm{mg} \mathrm{dm}^{-3}$. I sso se deve aos maiores teores de $\mathrm{P}_{\text {pre }}$ no solo, uma vez que se adicionaram fertilizantes na superfície sem posterior revolvimento do solo, saturando os sítios de mai or sorção. As isotermas de sorção de P no solo sob SPD mostraram-se menos inclinadas do que as sob SCC em baixas doses de P, cuja aplicação de uma mesma dose proporcionou maior aumento no P-solução no solo sob o primeiro sistema.

A disponibilidade deP mostrou-semuitosuperior no solo sob SPD do que sob SCC, mesmo em camadas mais profundas, pois o solo foi revolvido em 1989 para a incorporação do calcário, o que pode ter aumentado os teores de $\mathrm{P}$ nessas camadas. Essa maior disponibilidade, ou maior $\mathrm{P}_{\text {pre, }}$ alterou $\mathrm{O}$ traçado das isotermas. NoPVd, a $P_{\max }$, sem considerar o $P_{\text {pre, }}$ aumentou em camadas mais profundas. Com a inclusão do $P_{\text {pre, }}$, houve um incremento de $P_{\max }$ no solo sob SPD, na sucessão $A / M$ e nas duas camadas mais superficiais do solo (Quadro 1), refletindo os maiores teores de $\mathrm{P}$ disponível.
Latossolo Vermelho distroférrico típico

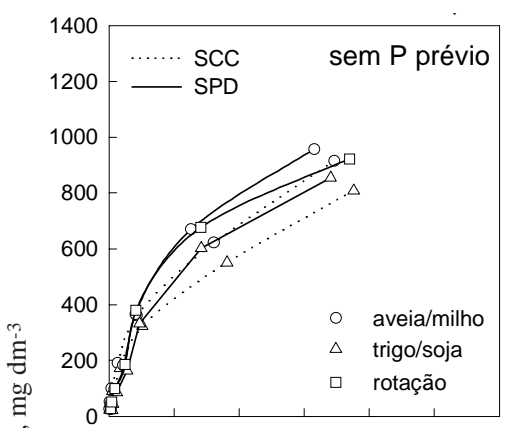

:

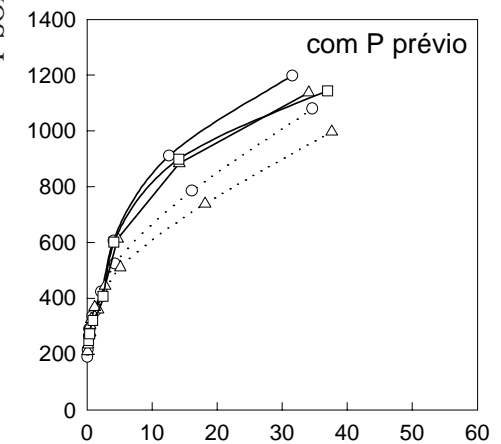

Latossolo Vermelho distrófico típico
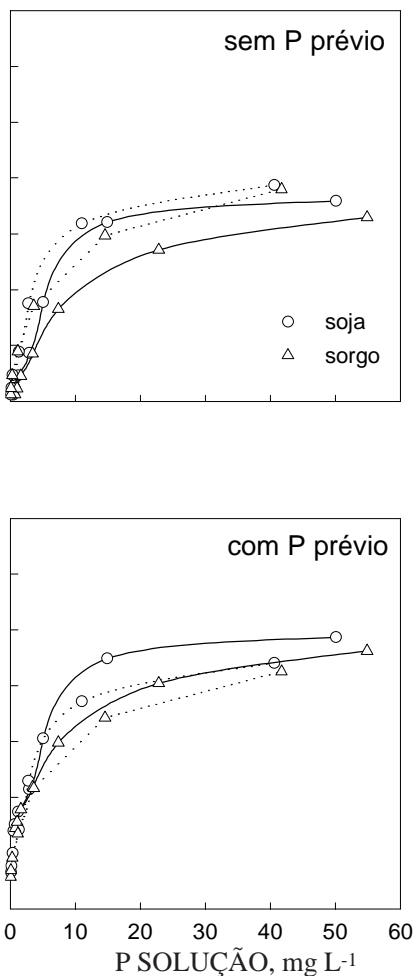

Argissolo Vermelho distrófico típico
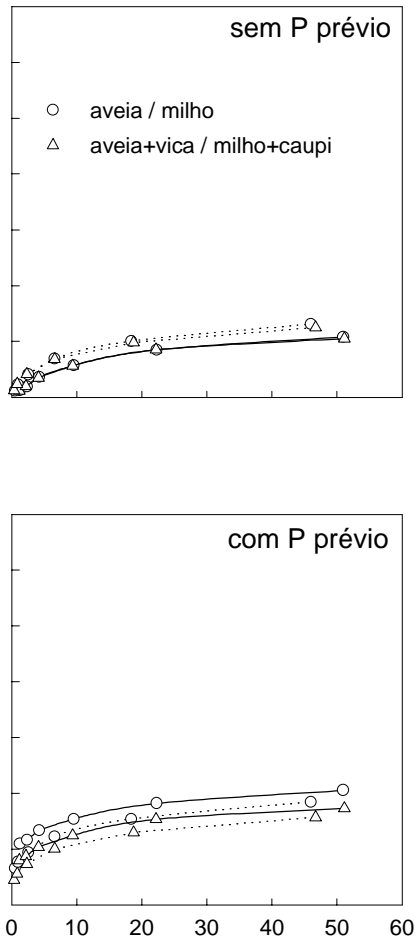

Figura 1. Relação entre o fósforo sorvido e o fósforo da solução da camada de 0-2,5 cm, sem e com a inclusão do fósforo previamente sorvido em diferentes solos, métodos de preparo e sucessões de culturas. 


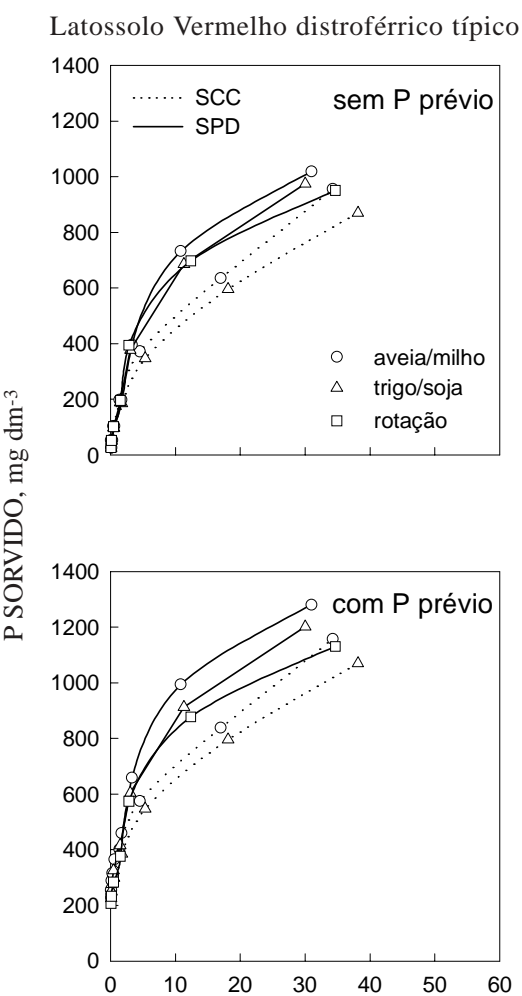

Latossolo Vermelho distrófico típico
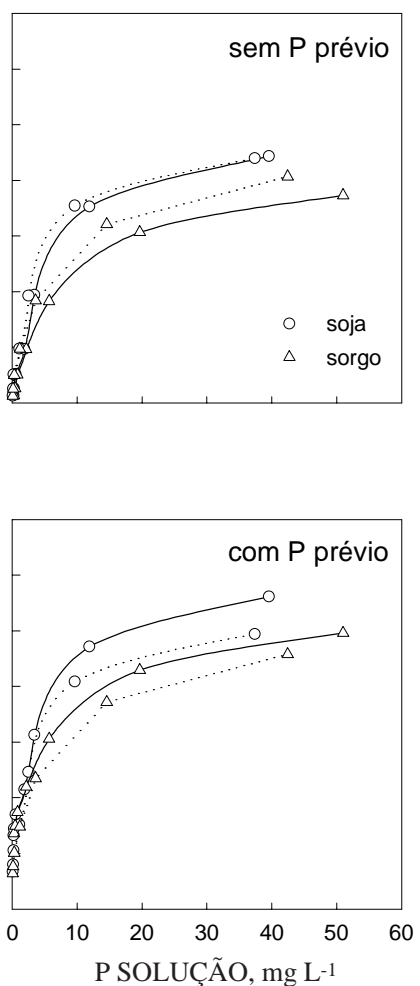

Argissolo Vermelho distrófico típico
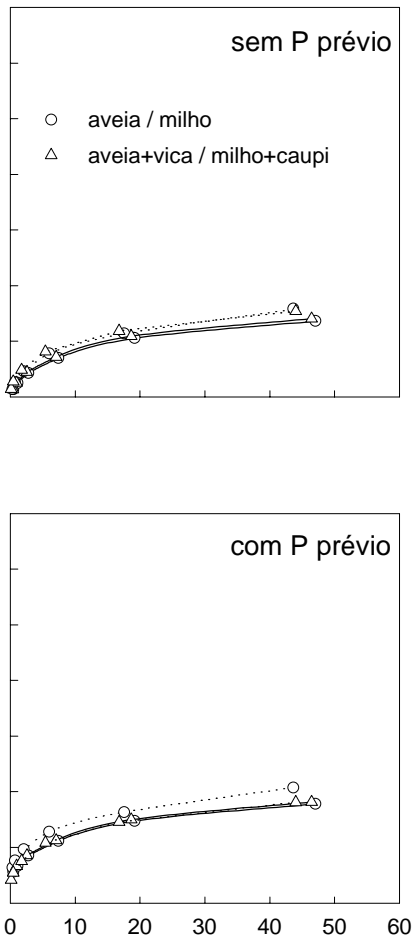

Figura 2. Relação entre o fósforo sorvido e o fósforo da solução da camada de 2,5-7,5 cm, sem e com a inclusão do fósforo previamente sorvido em di ferentes solos, métodos de preparo e sucessões de culturas.

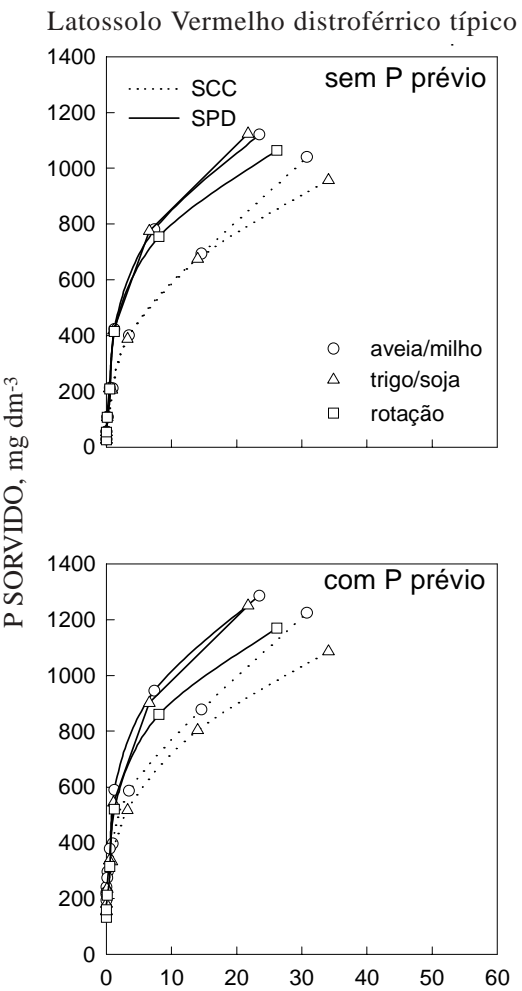

Latossolo Vermelho distrófico típico
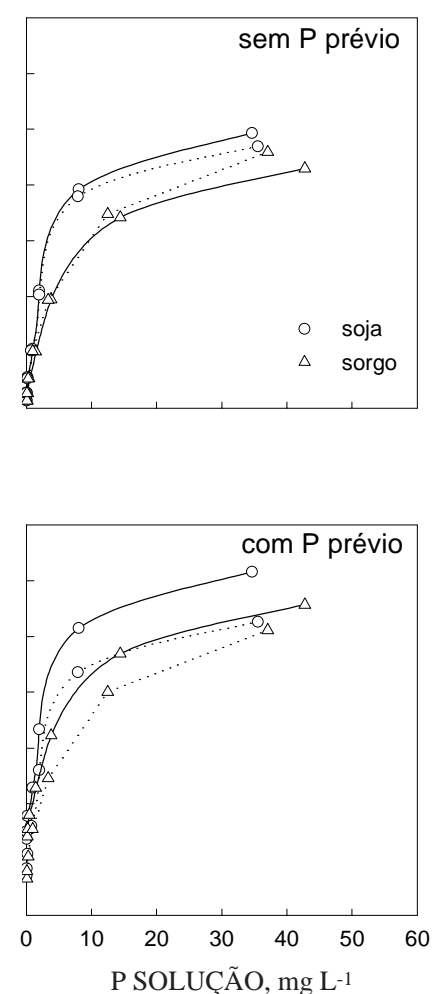

Argissolo Vermelho distrófico típico
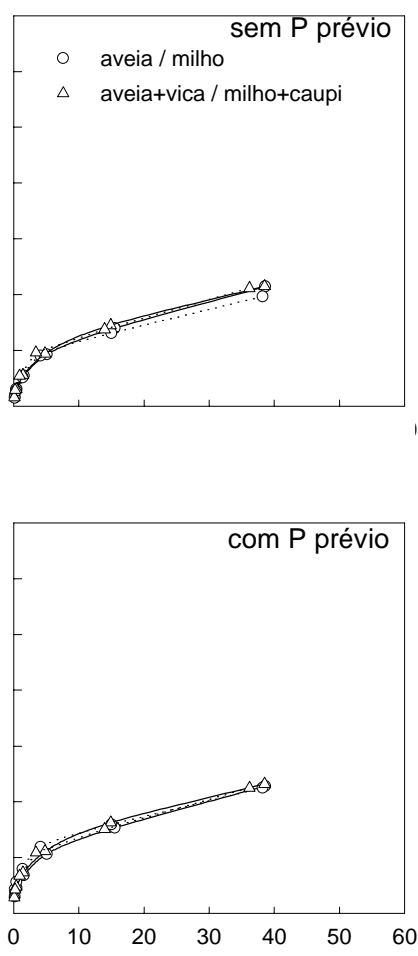

Figura 3. Relação entre o fósforo sorvido e o fósforo da solução da camada de 7,5-17,5 cm, sem e com a inclusão do fósforo previamente sorvido em diferentes solos, métodos de preparo esucessões de culturas. 
Quadro 1. Coefiecientes da equação de Langmuir para a sorção de fósforo em diferentes tipos de solo, métodos de preparo, sucessões de culturas e camadas amostradas, sem e com a inclusão do fósforo previamente adsorvido

\begin{tabular}{|c|c|c|c|c|c|}
\hline \multirow{2}{*}{ Solo(1) } & \multirow{2}{*}{ Fator } & \multicolumn{2}{|c|}{$P_{\text {max }}(4)$} & \multicolumn{2}{|c|}{$\kappa^{(5)}$} \\
\hline & & Sem ${ }^{(6)}$ & Com & Sem & Com \\
\hline & & 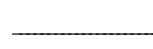 & - & 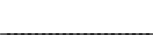 & {[} \\
\hline \multirow[t]{10}{*}{ LVdf } & Preparo do solo(2) & & & & \\
\hline & SPD & $1.191 \mathrm{Aa}$ & $1.195 \mathrm{Aa}$ & $0,214 \mathrm{Ab}$ & 0,783 Aa \\
\hline & $\begin{array}{l}\text { SCC } \\
\text { Sucessão(3) }\end{array}$ & $1.094 \mathrm{Ba}$ & $1.031 \mathrm{Ba}$ & $0,118 \mathrm{Ab}$ & $0,454 \mathrm{Ba}$ \\
\hline & $\mathrm{A} / \mathrm{M}$ & $1.203 \mathrm{Aa}$ & $1.165 \mathrm{Aa}$ & $0,160 \mathrm{Ab}$ & $0,481 \mathrm{Aa}$ \\
\hline & $\mathrm{T} / \mathrm{S}$ & $1.119 \mathrm{Ba}$ & $1.082 \mathrm{Ba}$ & $0,166 \mathrm{Ab}$ & $0,487 \mathrm{Aa}$ \\
\hline & Rotação & $1.118 \mathrm{Ba}$ & $1.154 \mathrm{Aa}$ & $0,234 \mathrm{Ab}$ & $0,467 \mathrm{Aa}$ \\
\hline & Profundidade, $\mathrm{cm}$ & & & & \\
\hline & $0-2,5$ & $1.159 \mathrm{Aa}$ & $1.098 \mathrm{Aa}$ & $0,091 \mathrm{Bb}$ & $0,321 \mathrm{Ba}$ \\
\hline & $2,5-7,5$ & $1.158 \mathrm{Aa}$ & $1.132 \mathrm{Aa}$ & $0,124 \mathrm{Bb}$ & $0,425 \mathrm{Ba}$ \\
\hline & $7,5-17,5$ & $1.141 \mathrm{Aa}$ & $1.159 \mathrm{Aa}$ & $0,312 \mathrm{Ab}$ & $0,783 \mathrm{Aa}$ \\
\hline \multirow[t]{9}{*}{ LVd } & Preparo do solo & & & & \\
\hline & SPD & $937 \mathrm{Ab}$ & $1.021 \mathrm{Aa}$ & $0,167 \mathrm{Bb}$ & 0,700 Aa \\
\hline & $\begin{array}{l}\text { SCC } \\
\text { Sucessão }\end{array}$ & $931 \mathrm{Aa}$ & $965 \mathrm{Ba}$ & $0,248 \mathrm{Ab}$ & $0,441 \mathrm{Ba}$ \\
\hline & Sorgo & $976 \mathrm{Aa}$ & $1.001 \mathrm{Aa}$ & $0,246 \mathrm{Ab}$ & $0,567 \mathrm{Aa}$ \\
\hline & Soja & $891 \mathrm{Bb}$ & $985 \mathrm{Aa}$ & $0,169 \mathrm{Bb}$ & $0,544 \mathrm{Aa}$ \\
\hline & Profundidade, $\mathrm{cm}$ & & & & \\
\hline & $0-2,5$ & $846 \mathrm{Cb}$ & $915 \mathrm{Ca}$ & $0,161 \mathrm{Bb}$ & $0,405 \mathrm{Ba}$ \\
\hline & $2,5-7,5$ & $937 \mathrm{Ba}$ & $989 \mathrm{Ba}$ & $0,191 \mathrm{Bb}$ & $0,527 \mathrm{ABa}$ \\
\hline & $7,5-17,5$ & $1.018 \mathrm{Aa}$ & $1.075 \mathrm{Aa}$ & $0,271 \mathrm{Ab}$ & $0,735 \mathrm{Aa}$ \\
\hline \multirow[t]{9}{*}{$P \vee d$} & Preparo do solo & & & & \\
\hline & & $354 \mathrm{Ab}$ & $390 \mathrm{Aa}$ & $0,114 \mathrm{Ab}$ & $0,444 \mathrm{Ba}$ \\
\hline & $\begin{array}{l}\text { SCC } \\
\text { Sucessão }\end{array}$ & $346 \mathrm{Aa}$ & $365 \mathrm{Ba}$ & $0,184 \mathrm{Ab}$ & $0,580 \mathrm{Aa}$ \\
\hline & $\mathrm{A} / \mathrm{M}$ & $353 \mathrm{Ab}$ & $388 \mathrm{Aa}$ & $0,133 \mathrm{Ab}$ & $0,552 \mathrm{Aa}$ \\
\hline & $A+V / M+C$ & $346 \mathrm{Aa}$ & $367 \mathrm{Aa}$ & $0,165 \mathrm{Ab}$ & $0,472 \mathrm{Aa}$ \\
\hline & Profundidade, $\mathrm{cm}$ & & & & \\
\hline & $0-2,5$ & $272 \mathrm{Cb}$ & $338 \mathrm{Ba}$ & $0,120 \mathrm{Ab}$ & $0,625 \mathrm{Aa}$ \\
\hline & $2,5-7,5$ & $324 \mathrm{Ba}$ & $347 \mathrm{Ba}$ & $0,154 \mathrm{Ab}$ & $0,570 \mathrm{Aa}$ \\
\hline & $7,5-17,5$ & $452 \mathrm{Aa}$ & $448 \mathrm{Aa}$ & $0,173 \mathrm{Ab}$ & $0,341 \mathrm{Ba}$ \\
\hline
\end{tabular}

1) LVdf = Latossolo Vermelho distroférrico típico; LVd = Latossolo Vermelho distrófico típico; PVd = Argissolo Vermelho distrófico típico. ${ }^{(2)}$ SPD = sistema plantio direto e SCC = sistema de cultivo convencional. ${ }^{(3)} \mathrm{A} / \mathrm{M}=$ aveia preta/milho; T/S =trigo/soja; Rotação =inverno (trigo, tremoço, colza e aveia preta +trevo) e verão (milho e soja); Sorgo e Soja = sorgo e soja antecedendo a col eta do sol o; $\mathrm{A}+\mathrm{V} / \mathrm{M}+\mathrm{C}=$ aveia preta + vica/milho + caupi. ${ }^{(4)} \mathrm{P}_{\max }=$ capacidade de sorção máxima. ${ }^{(5)} \mathrm{k}=$ energia de sorção. ${ }^{(6)}$ Sem e com a inclusão do fósforo previamente adsorvido.

Médias seguidas pela mesma letra, maiúscula na coluna e minúscula na linha, não diferem estatisticamente pelo teste Tukey a 5 \%.

O valor da constante relacionada com a energia de ligação ( $k$ ) aumentou com a inclusão do $P_{\text {pre }}$ no ajuste da isoterma de Langmuir. O valor médio de todos os tratamentos, no LVdf, passou de 0,166 para 0,618 $\mathrm{L} \mathrm{mg}^{-1}$; no LVd, de 0,207 para 0,570 $\mathrm{L} \mathrm{mg}^{-1}$, e no PVd, de 0,149 para 0,512 L mg ${ }^{-1}$, para oajuste sem ou com a inclusão do $\mathrm{P}_{\text {pre, }}$, respectivamente (Quadro 1). Os valores de $\mathrm{k}$ foram subestimados, uma vez que para a quantidade deP na solução de equilíbrio havia uma porção de $\mathrm{P}$ sorvido no solo que não foi computada no ajuste. I sso está de acordo com o modelo proposto por Parfitt et al. (1975), Parfitt (1978) e Ryden et al. (1977; 1987) em quea adsor ção ocorre em estádios. No estádio I, predomina a neutralização das cargas positivas, saturando-as quando todos os grupos $\mathrm{OH}_{2}{ }^{+}$forem compartilhados pel os íons fosfatos. No estádio II, ocorrem as trocas de ligantes e, no III, ocorrem interações de caráter mais físico com os íons Fejá octacoordenados, caracterizado pela baixa energia da interação. Então, adições subseqüentes de fosfatos tendem a diminuir a curvatura das isotermas de sorção (Barrow et al., 1998).

O $P_{\text {pre }}$ no solo LVDf perfez 21,18 e $11 \%$ da $P_{\text {max }}$, no SPD, e 18, 20 e $14 \%$, para as camadas de 0-2,5; 2,5-7,5 e 7,5-17,5 cm, respectivamente, não tendo diferenças quanto aos métodos de preparo e sucessões de culturas. No LVd, a contribuição do $P_{\text {pre }}$ foi de 29 e $12 \%$ da $P_{\text {max }}$, para o SPD e SCC, 
respectivamente, sendo levemente inferior nas camadas mais profundas. Nosolo PVd, o $P_{\text {pre }}$ perfez 39,18 e $5 \%$ da $P_{\max }$, sob SPD, e 27, 21 e $8 \%$ da $P_{\max }$, sob SCC, para as camadas 0-2,5, 2,5-7,5 e 7,5-17,5 cm, respectivamente (Quadro 2 ). Esses val ores estão de acordo com aqueles mencionados na literatura, variando de 17 a 66 \% da $\mathrm{P}_{\max }$ (Freese et al., 1992).

A contribuição percentual do $P_{\text {pre }}$ da camada superficial dos solos sob SPD foi inversamente proporcional ao seu teor de argila e óxidos de ferro. I sso está de acordo com o fato de que a magnitude da adsorção depende da quantidade de constituintes com capacidade de sorver moléculas neutras ou carregadas el etricamente. Nos solos de regiões tropicais esubtropicais, os óxidos deferro mal cristalizados, a quantidade dealumíniosubstituindoferro, os óxidos bem cristalizados e a caulinita são os responsáveis por esse fenômeno (Freese et al., 1992; Scheinost \& Schwertmann, 1995; Fontes \& Weed, 1996).

Deste modo, recomenda-se que o $\mathrm{P}_{\text {pre }}$ seja obtido diretamente com a dessorção por resina em membrana (Rheinheimer, 2000) e seja incluído no ajuste da isoterma de sorção.

\section{Sistemas de manejo de solos e os valores de $\mathbf{P}_{\text {max }}$ ek}

Nos três solos estudados, a adoção do SPD aumentou a $\mathrm{P}_{\max }$ em relação ao solo cultivado sob
SCC. Também a $P_{\max }$ aumentou nas camadas mais profundas dos três solos, sendo significativa para o LVd e PVd, cuja $P_{\text {max }}$ passou de 915 e $338 \mathrm{mg} \mathrm{dm}^{-3}$, na camada de $0-2,5 \mathrm{~cm}$, para 1.075 e $448 \mathrm{mg} \mathrm{dm}^{-3}$, na camada de 7,5-17,5 cm, respectivamente. As diferentes sucessões de culturas não afetaram a $P_{\max }$ e a k (Quadro 1).

Verificou-se que o teor de carbono orgânico foi mais el evado quando se utilizou oSPD até os $2,5 \mathrm{~cm}$ no LVdf; até os 7,5 cm, no LVd, e até os $2,5 \mathrm{~cm}$, no $\mathrm{PVd}$, com aveia/milho, e até os $7,5 \mathrm{~cm}$, com aveia + vica/milho + caupi (Quadro 3). Deste modo, não há relação entre o teor de carbono orgânico e a $P_{\max }$. Oloya \& Logan (1980) e Guertal et al. (1991) mostraram, que, em solo sob SPD, ocorre menor adsorção de fósforo na camada de $0-2 \mathrm{~cm}$ em relação às camadas mais profundas e ao SCC, determinado sem a inclusão do $P_{\text {pre. }}$ A quantidade de fósforo removida pela extração seqüencial com resina foi mais rápida e em maior quantidade na camada de 0-2 cm em relação às camadas de 2-6 e 6-8 cm. No entanto, após a extração, a capacidade de sorção de fósforo foi maior no SPD para todas as profundidades, especialmente para a camada superficial. Então, a menor sorção se deve ao maior teor de $P_{\text {pre }}$, saturando os sítios.

Os sol os queatual mente são manejados sob oSPD foram cultivados por vários anos sob oSCC. A adição

Quadro 2. Capacidade de sorção máxima $\left(P_{\max }\right)$ e fósforo previamente sorvido $\left(P_{\text {pre }}\right)$ em diferentes solos, métodos de preparos e profundidade de amostragem

\begin{tabular}{|c|c|c|c|c|c|}
\hline \multirow[t]{2}{*}{ Solo(1) } & \multirow[t]{2}{*}{ Preparo } & \multirow{2}{*}{$\begin{array}{c}\text { Profundidade } \\
\mathrm{cm}\end{array}$} & $P_{\max }{ }^{3}$ & \multicolumn{2}{|c|}{$\mathbf{P}_{\mathrm{pre}} \mathrm{e}^{(4)}$} \\
\hline & & & 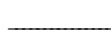 & - & $\%$ do $P_{\max }$ \\
\hline \multirow[t]{2}{*}{ LVdf } & $S P D^{(2)}$ & $\begin{array}{l}0-2,5 \\
2,5-7,5 \\
7,5-17,5\end{array}$ & $\begin{array}{l}1.182 \\
1.204 \\
1.200\end{array}$ & $\begin{array}{l}248 \\
223 \\
133\end{array}$ & $\begin{array}{l}21 \\
18 \\
11\end{array}$ \\
\hline & SCC & $\begin{array}{l}0-2,5 \\
2,5-7,5 \\
7,5-17,5\end{array}$ & $\begin{array}{r}972 \\
1.024 \\
1.099\end{array}$ & $\begin{array}{l}176 \\
202 \\
158\end{array}$ & $\begin{array}{l}18 \\
20 \\
14\end{array}$ \\
\hline \multirow[t]{2}{*}{ LVd } & SPD & $\begin{array}{l}0-2,5 \\
2,5-7,5 \\
7,5-17,5\end{array}$ & $\begin{array}{l}880 \\
851 \\
878\end{array}$ & $\begin{array}{l}261 \\
241 \\
250\end{array}$ & $\begin{array}{l}30 \\
28 \\
28\end{array}$ \\
\hline & SCC & $\begin{array}{l}0-2,5 \\
2,5-7,5 \\
7,5-17,5\end{array}$ & $\begin{array}{l}777 \\
842 \\
890\end{array}$ & $\begin{array}{r}99 \\
105 \\
109\end{array}$ & $\begin{array}{l}13 \\
12 \\
12\end{array}$ \\
\hline \multirow[t]{2}{*}{$P V d$} & SPD & $\begin{array}{l}0-2,5 \\
2,5-7,5 \\
7,5-17,5\end{array}$ & $\begin{array}{l}427 \\
454 \\
703\end{array}$ & $\begin{array}{r}165 \\
83 \\
32\end{array}$ & $\begin{array}{r}39 \\
18 \\
5\end{array}$ \\
\hline & SCC & $\begin{array}{l}0-2,5 \\
2,5-7,5 \\
7,5-17,5\end{array}$ & $\begin{array}{l}316 \\
362 \\
500\end{array}$ & $\begin{array}{l}86 \\
77 \\
42\end{array}$ & $\begin{array}{r}27 \\
21 \\
8\end{array}$ \\
\hline
\end{tabular}

(1) LVdf = Latossolo Vermelho distroférrico típico; LVd = Latossolo Vermelho distrófico típico; PVd = Argissolo Vermel ho distrófico típico. ${ }^{(2)} \mathrm{SPD}=$ sistema plantio direto e $\mathrm{SCC}=$ sistema de cultivo convencional. ${ }^{\left({ }^{3}\right)} \mathrm{P}_{\max }=$ capacidade de sorção máxima. ${ }^{(4)} \mathrm{P}_{\text {pre }}=$ fósforo previamente sorvido. 
Quadro 3. Teores de carbono orgânico em diferentes ti pos de solos, métodos de preparo do solo, sucessões de culturas e camadas amostradas

\begin{tabular}{|c|c|c|c|c|c|}
\hline \multirow{2}{*}{$\begin{array}{c}\text { Profundidade } \\
\mathrm{cm}\end{array}$} & \multicolumn{2}{|c|}{ SCC(1) } & \multicolumn{2}{|c|}{ SPD } & \\
\hline & $\ldots$ & 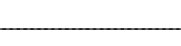 & $\mathrm{g} \mathrm{dm}^{-3}$ & 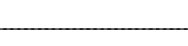 & - \\
\hline \multicolumn{6}{|c|}{ Latossolo Vermel ho distroférrico típico } \\
\hline & $A / M^{(2)}$ & $T / S$ & $\mathrm{~A} / \mathrm{M}$ & $\mathrm{T} / \mathrm{S}$ & Rotação \\
\hline $\begin{array}{l}0,0-2,5 \\
2,5-7,5 \\
7,5-17,5\end{array}$ & $\begin{array}{l}20,8 \\
24,6 \\
23,9\end{array}$ & $\begin{array}{l}22,2 \\
25,8 \\
23,5\end{array}$ & $\begin{array}{l}25,8 \\
22,6 \\
21,2\end{array}$ & $\begin{array}{l}27,4 \\
23,2 \\
20,5\end{array}$ & $\begin{array}{l}26,6 \\
23,0 \\
20,9\end{array}$ \\
\hline \multicolumn{6}{|c|}{ Latossolo Vermel ho distrófico típico } \\
\hline & Sorgo & Soja & Sorgo & Soja & \\
\hline $\begin{array}{l}0,0-2,5 \\
2,5-7,5 \\
7,5-17,5\end{array}$ & $\begin{array}{l}18,7 \\
19,0 \\
21,1\end{array}$ & $\begin{array}{l}19,7 \\
21,1 \\
21,2\end{array}$ & $\begin{array}{l}33,1 \\
27,3 \\
21,7\end{array}$ & $\begin{array}{l}32,9 \\
27,7 \\
22,7\end{array}$ & \\
\hline \multicolumn{6}{|c|}{ Argissolo Vermelho distrófico típico } \\
\hline & $\mathrm{A} / \mathrm{M}$ & $A+V / M+C$ & $\mathrm{~A} / \mathrm{M}$ & $A+V / M+C$ & \\
\hline $\begin{array}{l}0,0-2,5 \\
2,5-7,5 \\
7,5-17,5\end{array}$ & $\begin{array}{l}15,6 \\
16,4 \\
17,1\end{array}$ & $\begin{array}{l}17,5 \\
18,6 \\
17,7\end{array}$ & $\begin{array}{l}24,2 \\
16,7 \\
16,7\end{array}$ & $\begin{array}{l}32,1 \\
20,0 \\
17,6\end{array}$ & \\
\hline
\end{tabular}

(1) SCC = sistema de cultivo convencional e SPD = sistema plantio direto. ${ }^{(2)} \mathrm{A} / \mathrm{M}$ = aveia preta/milho; T/S = trigo/soja; Rotação = inverno (trigo, tremoço, colza e aveia preta + trevo) e verão (milho e soja); Sorgo e Soja = sorgo e soja antecedendo a col eta do solo; $\mathrm{A}+\mathrm{V} / \mathrm{M}+\mathrm{C}=$ aveia preta + vica/milho + caupi.

defertilizantes fosfatados nesse sistema tinha etem de atender às exigências nutricionais das culturas e compensar os fenômenos de sorção e erosão. Deste modo, os sítios de maior sorção de fosfato são saturados pela el evação do $\mathrm{pH}$ do sol o e pel o próprio fosfato. A adsorção do fósforo aumenta as cargas negativas semipermanentes e diminui o potencial elétrico das partículas coloi dais, onde o fósforo migra lentamente para o interior dos óxidos (Barrow et al., 1998), o que justifica a menor adsorção do fosfato adicionado posteriormente (Raven \& H ossner, 1993), como observado neste trabal ho.

A $k$ foi maior no SPD do que no SCC nos Latossolos e menor no Argissolo. Também nos Latossolos, os valores da $\mathrm{k}$ das duas primeiras camadas foram menores do que os da de camada 7,5-17,5 cm, enquanto, noArgissolo, a k foi maior na camada de 0-2,5 cm (Quadro 1).

Nos Latossolos, houve correlação negativa entre os valores de $\mathrm{k}$ e os teores de carbono orgânico do solo para o SPD. Vários autores têm evidenciado que a matéria orgânica diminui a área superficial dos colóides inorgânicos e a adsorção de fósforo (Haynes, 1984; Sibanda \& Young, 1986). O incremento no teor de matéria orgânica pode aumentar a disponibilidade deste nutriente por diminuir a "energia de ligação" do fosfato com os colóides inorgânicos sem alterar a $\mathrm{P}_{\max }$ (Quadro 1). I sso explicaria o porquê do aumento no potencial de dessorção do fósforo nativo quando da aplicação de resíduos vegetais em sol o com baixa disponibilidade de fósforo e alta capacidade de adsorção (Haynes, 1984; Haggar et al., 1991) e da mel horia na eficiência dos fertilizantes fosfatados (Hue, 1991).

No entanto, no caso do Argissolo, à medida que aumentou o teor de carbono orgânico, também aumentou a "energia de ligação" do fosfato com o sol o cultivado sob SPD. Considerando que esses sol os apresentam baixos teores de argila e óxidos deferro, que foram adicionadas grandes quantidades de fosfatos na camada superficial, que foi adicionado calcário na superfície do solo e que as isotermas não permitem separar os fenômenos responsáveis pelo desaparecimento do fósforo da solução, acredita-se que tenha ocorrido a formação de precipitados de fosfatos de cál cio.

\section{CONCLUSÕES}

1. O ajuste de isotermas sem considerar a quantidade de fósforo sorvida pelo solo leva a interpretações errôneas. A sua inclusão no modelo de Langmuir aumentou, em média, 2,9 vezes o val or da constante relacionada com a energia de ligação, não alterou a capacidade de sorção máxima de fósforo no Latossolo Vermel ho distroférrico típico e 
aumentou na camada superficial do Latossolo Vermel ho distrófico típico e do Argissolo Vermelho distrófico típico.

2. O fósforo previamente sorvido pela camada de $0-2,5 \mathrm{~cm}$ sob sistema plantio direto representa 21 , 30 e 39 \% da capacidade de sorção máxima do solo, LVdf, LVd e PVd, respectivamente.

3. A capacidade de sorção máxima de fósforo, determinada com a inclusão do fósforo previamente sorvido pel o solo, é pouco influenciada pel os métodos de preparo do solo, sucessões de culturas e camadas amostradas.

\section{LITE RATURA CITADA}

AMADO, T.J .C.; MIELNICZUK, J .; FERNANDES, S.B. \& BAYER, C. Culturas de cobertura, acúmulo de nitrogênio total no solo e produtividade de milho. R. Bras. Ci. Solo, 23:679686, 1999.

BARROW, N.J. The descrition of phosphate adsortion curves. J . Soil Sci., 29:447-462, 1978.

BARROW, N.J .; BOLLAND, M.D.A. \& ALLEN, D.G. Effect of additions of superphosphate on sorption of phosphate. Aust. J. Soil Res., 36:359-372, 1998.

DALLA ROSA, A.; MERTEN, G.H. \& MIELNICZUK, J. Produtividade do trigo e da soja em sistemas de culturas e preparos de solo. Trigo Soja, 113:14-18, 1991.

FONTES, M.P.F. \& WEED, S.B. Phosphate adsorption by clays from Brazilian Oxisols: relationships with specific surface area and mineralogy. Geoderma, 72:37-51, 1996.

FOX, R.L. \& KAMPRATH, E.J. Phosphate sorption isotherms for evaluating the phosphate requirements of soils. Soil Sci. Soc. Am. Proc., 34:902-906, 1970.

FREESE, D.; van der ZEE, S.E.A.T.M . \& van RIEMSDIJ K, W.H. Comparison of different models for phosphate sorption as a function of the iron and aluminium oxides of soils. J. Soil Sci., 43:729-738, 1992.

GUERTAL, E.A.; ECKERT, D.J .; TRAINA, S.J . \& LOGAN, T.J . Differential phosphorus retention in soil profiles under notill crop production. Soil Sci. Soc. Am. J., 55:410-413, 1991.

HAGGAR, J.P.; WARREN, G.P.; BEER, J.W. \& KASS, D. Phosphorus availability under alley cropping and mulched and unmulched sole cropping systems in Costa Rica. Plant Soil, 137:275-283, 1991.

HARTIKAINEN, H. Potencial mobility of accumulated phosphorus in soil as estimated by the indices of Q/l plots and by extractant. Soil Sci., 152:204-209, 1991.

HAYNES, R.J . Lime and phosphate in the soil-plant systems. Adv. Agron, 37:249-315, 1984.

HUE, N.V. Effects of organic acids/anions on P sorption and phytoavailability in soil with different mineralogies. Soil Sci., 152:463-471, 1991.
McBRIDE, M.B. Environmental chemistry of soils. New York, Oxford University Press, 1994. 406p.

MESQUITA, M.V. \& TORRENT,J . Phosphatesorption as related to mineralogy of a hydrosequence of soils from the Cerrado region (Brazil). Geoderma, 58:107-123, 1993.

MURPHY, J.\& RILEY, J.P. A modified single solution method for the determination of phosphatein natural waters. Anal. Chem. Acta, 27:31-36, 1962.

OLOYA, T.O. \& LOGAN, T.J. Phosphate desorption from soils and sediments with varying levels of extractable phosphate. J. Environ. Qual., 9:526-531, 1980.

PARFITT, R.L. Phosphate reactions with natural allophane, ferrihydrite and goethite. J. Soil Sci., 40:359-369, 1989.

PARFITT, R.L. Anion adsorption by soils and soil materials. Adv. Agron, 30:1-50, 1978.

PARFITT, R.L.;ATKINSON, R.J . \& SMART, R.C. The mechanism of phosphate fixation by iron oxides. Soil Sci. Soc. Am. J., 39:837-841, 1975.

RAVEN, K.P. \& HOSSNER, L.R. Phosphorus desorption quantity-intensity relationships in soil. Soil Sci. Soc. Am. J., 57:1501-1508, 1993.

RHEINHEIMER, D.S. Dinâmica do fósforo em sistemas de manejo de solos. Porto Alegre, Universidade Federal do Rio Grande do Sul, 2000. 210p. (Tese de Doutorado)

RHEINHEIMER, D.S.; ANGHINONI, I. \& KAMINSKI, J. Depleção do fósforo inorgânico de diferentes frações provocada pela extração sucessiva com resina em diferentes solos e manejos. R. Bras. Ci. Solo, 24:345-354, 2000.

RHEINHEIMER, D.S. \& ANGHINONI,I. Distribuição do fósforo inorgânico em sistemas de manejo de solo. Pesq. Agropec. Bras, 36:151-160, 2001.

RYDEN, C.J .; MCLAUGHLIN, J.R. \& SYERS, J.K. Mechanisms of phosphate sorption by soils and hydrous ferric oxide gel. J. Soil Sci., 28:72-92, 1977.

RYDEN, C.J .; SYERS, J.K. \& TILLMAN, R.W. I norganic anion sorption and interactions with phosphate sorption by hydrous ferric oxide gel. J. Soil Sci., 38:211-217, 1987.

SCHEINOST,A.C. \& SCHWERTMANN, U. Predicting phosphate adsorption-desorption in a soilscape. Soil Sci. Soc. Am. J., 59:1575-1580, 1995.

SELLES, F.; KOCHHANN, R.A.; DENARDIN, J .E.; ZENTNER, R.P. \& FAGANELLO, A. Distribution of phosphorus fractions in Brazilian Oxisol under different tillagesystems. Soil Till. Res., 44:23-34, 1997.

SIBANDA, H.M. \& YOUNG, S.D. Competitive adsortion of humic acids and phosphate on goethite, gibbsite and two tropical soils. J. Soil Sci., 37:197-204, 1986.

TOLNER, L. \& FÜLEKY, G.Y. Determination of the originally adsorbed soil phosphorus by modified Freundlich isotherm. Comm. Soil Sci. Plant Anal., 26:1213-1231, 1995. 